\title{
COEXISTENCE OF TUBERCULOSIS AND ADENOCARCINOMA OF THE COLON
}

\author{
Singh $\mathrm{Y}^{*}$, Sayami $\mathrm{G}^{\star}$, Khakurel $\mathrm{M}^{\star}$
}

\section{ABSTRACT}

A 60-years-old, male patient presented with a-tender swelling in the right iliac fossa with right hip flexion deformity suggesting of psoas abscess. Following emergency drainage of faeculant pus, the general condition of the patient improved. The barium enema showed leaking ascending colonic ulcer and colonoscopic examination revealed nonspecific ulcerative lesion in the ascending colon. Repeat investigations were also non-conclusive except there was a stricture at the ascending colon on colonoscopy. A second operation showed that there was a huge mass of terminal ileum, cecum and ascending colon and perforation of ascending colonic ulcer most likely carcinoma. Right hemicolectomy was performed with uneventful postoperative period. Histopathological examination revealed well differentiated adenocarcinoma limited to the muscle layer of the ascending colon and features suggestive of colonic tuberculosis at the same site. Antitubercular treatment was completed with regular follow-up and monitoring on CEA level. Patient has been doing well 3 years postoperatively. Although rare, the coexistence of colonic tuberculosis with carcinoma may occur. A definitive diagnosis can be established only by histological examination.

\section{Key Words: Colonic tuberculosis, Adenocarcinoma, Histology.}

\section{INTRODUCTION}

Tuberculosis (TB) has been regarded as a global health problem and is more common in the developing world. ' Although gastrointestinal $\mathrm{TB}$ is one of the common forms of extra pulmonary $\mathrm{TB}$, colonic $\mathrm{TB}$ is less common. It is seen that chronic inflammatory bowel diseases have been associated with an increased risk of malignancy. ${ }^{3-5}$ However, the coexistence of intestinal TB and adenocarcinoma has rarely been described in English literature. ${ }^{6-9}$ Because of the rarity of the disease, many aspects of this occurrence remain to be elucidated. Herein we report a case of colonic TB coexisting with adenocarcinoma of the ascending colon, unusually presenting as a right psoas abscess and review the related literature.

\section{CASE REPORT}

The patient was 60-years-old, male of middle class family, a government officer who was admitted to the Department of Surgery, Tribhuvan University Teaching Hospital, Kathmandu, Nepal with a history of 2 months aching right iliac fossa swelling, anorexia and weight loss. He had also a history of high grade fever and unable to extend his right lower limb for one week prior to presentation. His past medical and family history were not contributory to the present illness. On clinical examination, there was a tender swelling in the right iliac fossa with right hip flexion deformity. An abdominal ultrasound also revealed right psoas abscess. Chest and spine radiographs were normal. An emergency drainage of about $100 \mathrm{ml}$ feculent

* Tribhuvan University Teaching Hospital, Kathmandu, Nepal.

Address for correspondence : Dr. Yogendra Singh

Department of Surgery

GPO Box No.: 5204, Kathmandu, Nepal

Email: yp_singh@hotmail.com

INMA .lanıarv - Fehruary 200342 
pus was done and then the general condition of the patient was improving except having a few spikes of fever. The barium enema showed leaking ascending colonic ulcer and colonoscopic examination revealed nonspecific ulcerative lesion in the ascending colon. Repeat investigations were also non-conclusive except there was a stricture at the ascending colon on colonoscopic examination. A second operation showed that there was a $20 \times 15 \mathrm{~cm}$ mass of terminal ileum, cecum and ascending colon and perforation of ascending colonic ulcer most likely carcinoma. Right hemicolectomy was performed and the postoperative period was uneventful. Histopathological examination revealed well differentiated tubular adenocarcinoma limited to the muscle layer of the ascending colon (Fig. 1) and features suggestive of colonic

Fig. 1

Fig. 1: Well differentiated tubular adenocarcinoma infiltrating into the muscular layer of the colon (HEX 40)

TB at the same site (Fig. 2). One year antitubercular treatment with Isoniazid (INH), Rifampicin, Ethambutol and

\section{Fig. 2}

\section{Fig.2: Epitheloid cell granuloma with Langerhans' giant cell (HEX20)}

Pyrizinamide for first 2 months, and then INH and Rifampicin for another 10 months was completed. The patient has been in regular follow-up and monitoring on CEA level. He has been doing well and no evidence of detectable recurrence of tuberculosis or cancer at a follow-up of 3 years postoperatively.

\section{DISCUSSION}

The gastrointestinal $\mathrm{TB}$ in much more common in the developing countries than in the West. ${ }^{1}$ Ileocecal TB is the commonest involvement of the gastrointestinal tract. ${ }^{10}$ Although there is no characteristic colonoscopic finding for intestinal TB, colonoscopic biopsy has proven value for the diagnosis of TB. ${ }^{11}$ intestinal TB occurring together with colorectal cancer is an uncommon event and presents an interesting patho-etiological relationship.

The exact association of intestinal TB and carcinoma is not well established but the possibility of cause and effect relationship does exist. ${ }^{12}$ It is still not clear whether the presence of disease triggers the other. However, it is speculated that $\mathrm{TB}$, especially long standing untreated tubercular colitis might initiate cancer, by a process of carcinogenesis similar to one in Crohn's disease or schistosomiasis. ${ }^{13}$ Furthermore, a decreased incidence of colorectal carcinomas was found in the patients with pulmonary TB compared to those who had not. ${ }^{14}$ This coexistence may be a chance on one hand or an immunity to tubercle bacilli might be associated with decreased susceptibility to colorectal carcinoma.

The coexistence of the carcinoma and tuberculosis in the ileocecal region, the commonest site for intestinal TB, supports the hypothesis that tuberculosis precedes carcinoma. Furthermore, the most important histological finding is that of the carcinoma, which shows a well differentiated adenocarcinoma with a tendency to produce mucin. ${ }^{15}$ This unique finding is consistent with our case. This finding may support the possibility that the cancer originated from a tubercular lesion. As yet to be studied in detail, colonic TB might increase the risk of carcinoma by producing mucosal dysplasia.

Like in our case, the patient should receive anti-tubercular therapy postoperatively to prevent a relapse of TB. ${ }^{7}$ However, adjuvant cancer chemotherapy is not a well accepted practice in all patients with colorectal carcinoma. If it is necessary to give anti-mitotic as well as antitubercular therapy, the regimen would be cautiously selected and well-supervised. Because, there is a chance of flare-up of TB due to cancer chemotherapy induced immunosuppression.

\section{REFERENCES}

1. Raviglione MC, Snider D, Kochi A. Global epidemiology of tuberculosis. J AMA 1995; 273: 220-6.

2. Marshall J B. Clinical review: tuberculosis of the gastrointestinal tractand peritoneum. AmJ Gastroenterol 1993; 88: 989-97. 
3. WeedonDD, ShorterRG, IlstrupDM, Huizenga KA and Taylor WF. Crohn's disease and cancer. NEnglJ Med 1973; 289: 1099-1103.

4. GreensteinA , SacharDB, Smith H, J anowitz HD, and Aufses AHJ r. Pattems of neoplasia in Crohn's disease and ulcerative colitis. Cancer 1980; 46: 403-407.

5. HamiltonSR. Colorectal carcinoma in patients with Crohn's disease. Gasteroenterol 1985; 89: 398-407.

6. Randall KJ , and Spalding J E. Simultaneous carcinoma and tuberculosis of the colon: report of a case and review of the literature. BrJ Surg 1946; 33: 372-5.

7. Leong AFPK, Seov-Choen F, and GohHS. Colorectal cancer and intestinal tuberculosis. Ann Acad Med Singapore 1993; 22: 934-6.

8. Maheshwari V, Alam K, Indu, and Tyagi SP. Ileocecal tuberculosis associated with adenocarcinoma of the caecum and colon. J Indian MedAssoc 1995; 93: 392-3.

9. Isaacs P, Zissis M. Colonic tuberculosis and adenocarcinoma: an unusual presentation. EurJ Gastroenterol Hepatol 1997; 9: 913-5.
10. Hsiao T-J, Wong J -M, Shieh $M-J$, Wu C-H and Wang C-Y. Colonofiberoscopic diagnosis of intestinal tuberculosis. J Formos Med Assoc 1998; 97: 21-5.

11. Kalvavia I, KottlerRE, Marks IN. The role of colonoscopy in the diagnosis of tuberculosis. J din Gastroenterol 1988; 10: 16-23.

12. J ain BK, ChandraSS, NarasimhanR, AnanthakrishnariN and Mehta RB. Coexisting tuberculosis and carcinoma of the colon. Aust N ZJ Surg 1991; 61: 828-31.

13. Mishra MC, KapoorVK, Sharma LK. Co-existing carcinoma and tuberculosis of the colon. QJ SurgSci 1985; 21: 46-9.

14. Goulston E. Colorectal cancer-tuberculosis connection. Med J Aust 1981; 1: 637-8.

15. TanakaK, KondoS, HattoriF, etal. A case ofcolonic carcinoma associated with intestinal tuberculosis, and ananalysis of 26 cases reported in J apan. Gan No Rinsho 1987; 33:1117-23. 